\title{
Characterization and expression profile of CaNAC2 pepper gene
}

\section{Wei-Li Guo ${ }^{1,2 t}$, Shu-Bin Wang ${ }^{3 t}$, Ru-Gang Chen ${ }^{1+}$, Bi-Hua Chen², Xiao-Hua Du², Yan-Xu Yin ${ }^{1}$, Zhen-Hui Gong ${ }^{*}$ and Yu-Yuan Zhang ${ }^{2}$}

${ }^{1}$ College of Horticulture, Northwest A\&F University, Yangling, China, ${ }^{2}$ School of Horticulture Landscape Architecture, Henan Institute of Science and Technology, Xinxiang, China, ${ }^{3}$ Institute of Vegetable Crops, Jiangsu Academy of Agricultural

Sciences, Nanjing, China

The plant-specific NAC (NAM, ATAF, and CUC) transcription factors have diverse role in development and stress regulation. A new transcript encoding NAC protein, homologous to nam-like protein 4 from Petunia was identified from an ABA-regulated subtractive cDNA library of Capsicum annuum seedling. Here, this homolog (named CaNAC2) from C. annuum was characterized and investigated its role in abiotic stress tolerance. Our results indicated that a plant-specific and conserved NAC domain was located in the N-terminus domain of CaNAC2 which was predicted to encode a polypeptide of 410 amino acids. Phylogenetic analysis showed that CaNAC2 belonged to the NAC2 subgroup of the orthologous group 4d. The protein CaNAC2 was subcellularly localized in the nucleus and it had transcriptional activity in yeast cell. CaNAC2 was expressed mainly in seed and root. The transcription expression of CaNAC2 was strongly induced by cold, salt and ABA treatment and inhibited by osmotic stress and SA treatment. Silence of CaNAC2 in virus-induced gene silenced pepper seedlings resulted in the increased susceptibility to cold stress and delayed the saltinduced leaf chlorophyll degradation. These results indicated that this novel CaNAC2 gene might be involved in pepper response to abiotic stress tolerance.

Keywords: Capsicum annuum L., CaNAC2, expression profile, abiotic stress, virus-induced gene silencing

\section{Introduction}

Pepper (Capsicum annuum L.), the Solanaceae family, is an important spicy crop. Pepper plants originate from tropical regions and the optimal temperature for its growth ranges between 21 and $27^{\circ} \mathrm{C}$ (Deng et al., 2009). Low temperature severely affects the growth and reproduction of pepper plants, resulting in economic losses (Pressman et al., 2006; Korkmaz et al., 2010; Schwarz et al., 2010). Cold stress has created a great threat to the vulnerability of pepper. To respond to low temperature stress, plants have caused many physiological, and biochemical modifications to adapt to stress condition (Thomashow, 1999; Shinozaki et al., 2003). Such changes are intricately regulated by genes $t$. Among these regulating genes, plant transcription factors play an important role in the activation of genes related with stress tolerance.

Plant-specific NAC transcription factors comprise a large gene family. A highly conserved $\mathrm{N}$-terminal of these family proteins contains five sub-domains (A-E) and has the DNA binding ability of NAC proteins. The C-terminal section of NAC proteinsis diverged and potentially activates transcription (Ernst et al., 2004). A number of studies have revealed plant NAC proteins to be involved in transcriptional regulation in various processes. Some are related to the development 
of the shoot apical meristem, lateral root formation, senescenceand secondary wall formation (Xie et al., 2000; Duval et al., 2002; Olsen et al., 2005). Other NAC proteins have been implicated in response to various abiotic stresses, including abscisic acid (ABA), ethylene, drought, high salt, and low temperature (Tran et al., 2004; He et al., 2005; Hu et al., 2008). In Arabidopsis, the expression of ANAC019, ANAC055, or ANAC072 is induced by ABA, drought, salinity or cold stress and over expression of these genes in transgenic plants confers a constitutive increase in stress tolerance (Fujita et al., 2004; Tran et al., 2004). Arabidopsis AtNAC2 is also induced by salinity, ABA, and it has been predicted to be involved in the ethylene and auxin signaling pathways as a down-stream gene (He et al., 2005). In rice, some NAC genes have been characterized to be involved in responses to environmental stimuli (Hu et al., 2006, 2008; Jeong et al., 2010). More recently, in the Solanaceae family, Solanum tuberosum StNAC gene isup-regulated by Phytophtora infestans infection (Collinge and Boller, 2001), C. annuum CaNAC1 gene is induced by bacterial pathogen and SA treatment (Oh et al., 2005), and SINAC1 and SINAM1 genes from S. lycopersicum are up-regulated by salinity (Yang et al., 2011) and another SINAC3 is down-regulated by drought, salinity and ABA (Han et al., 2012). Therefore, pepper NAC transcription factors associated with stress tolerance particularly need further to be investigated.

In order to divulge the mechanism involved in cold-tolerance, suppression subtractive hybridization ( $\mathrm{SSH}$ ) revealed that ABAmediated candidate genes have been fully identified in pepper seedlings subjected to cold stress (Guo et al., 2013). One of the genes cloned from the forward subtraction was homologous to Petunia nam-like protein 4 encoding NAC transcription factor. Expression of this NAC homolog was significantly induced by cold stress and ABA. However, the involvement of this gene in the stress tolerance remains to be investigated. Here, we reported the characterization of a novel pepper NAC transcription factor gene designated as CaNAC2. Expression profiles in response to abiotic stress, and ABA, SA treatment were also examined by quantitative real time-PCR (qRT-PCR). Furthermore, the function of this gene silencing in pepper plants was studied by a virus-induced gene silencing (VIGS) method. These findings suggest that CaNAC2 in pepper could be involved in defense response against abiotic stress.

\section{Results}

\section{Cloning and Sequence Analysis of CaNAC2 Gene}

Pepper cold-related candidate genes from SSH library was reported previously (Guo et al., 2013). One of the isolated clones showed $82 \%$ homologous to nam-like protein 4 from Petunia. This homolog was used as the initial probe to screen the Solanaceae EST database in GenBank and the full-length clone was isolated by a homology-based candidate gene method. We designated it CaNAC2 followed with CaNAC1 that had been functionally identified in C. annuum (Oh et al., 2005). The entire cDNA fragment was $1,490 \mathrm{bp}$ in length and contained a single open reading frame (ORF) of 1,230 bp (GenBank accession No. JX402928, Supplementary Figure S1). It encoded 409 amino acids with $45.80 \mathrm{kDa}$ molecular mass and a theoretical isoelectric point of 5.34 .

A phylogenetic tree was conducted between the overall amino acid sequences of CaNAC2 and other known NAC-domain proteins. CaNAC2 was clustered into the NAC2 subgroup (Figure 1) and highly homologous to NH4 protein from Petunia (84\%, identity). Whereas, CaNAC2 was low homologous to ANAC053, ANAC050, and NAC2 from Arabidopsis, with similarity of 30, 42, and 26\%, respectively (data not shown).

To further analyze the NAC domain of CaNAC2, the overall amino acid sequences of NAC protein were analyzed for alignment. Result showed that the N-terminal region of CaNAC2 contained a conserved NAC domain (29-200 aa) and was divided into five sub-domains (A-E), while the C-terminal was a diversified transcription regulatory region (TRR; Supplementary Figure S2). A putative nuclear localization signal (NLS) was predicted at the N-terminus (132-158 aa). The C-terminal regions contained several putative a-helical motifs that did not form transmembrane motifs, structurally distinct from NTLs (NAC with transmembrane motif 1-like; Kim et al., 2007).

\section{Subcellular Localization and Transcription Activation Assay of CaNAC2 Protein}

CaNAC2 was predicted to be nucleus localized by ProComp v 8.0 program. To confirm this prediction, the subcellular localization of CaNAC2-GFP fusion protein was demonstrated by fusing the entire CaNAC2 coding sequence to GFP and expressed them under the control of the CaMV35S promoter in the onion epidermal cells. Cells expressing GFP alone displayed diffuse cytoplasmic and nuclear staining (Figure 2A) supported by Guo et al. (2015), while the GFP-CaNAC2 full length protein was located only in the nucleus and the 132-158 amino acids containing the putative NLS can mediate the nuclear targeting of the protein.

To validate that CaNAC2 functions as a transcriptional activator, the ORF of CaNAC2 was fused to the GAL4 DNA binding domain (GAL4-DB) in the vector pGBKT7 and the construct was transformed into yeast strain AH109 (Figure 2B). Colonies transformed with $\mathrm{pBD}-\mathrm{CaNAC2}$ construct grew on both SD/-Leu/-Trp and SD/-Ade/-His/-Leu/-Trp unlike the vector-transformed colonies, indicating that the CaNAC2 had transcriptional activity in yeast cell.

\section{Expression Patterns of CaNAC2 Under Abiotic Stresses}

The expression pattern of CaNAC2 in different pepper tissues were detected by qRT-PCR. This analysis revealed that the transcription of CaNAC2 was abundant in root and seed, low in stem, leaf, flower, and fruit (Figure 3A).

The transcription expression of CaNAC2 was further investigated by abiotic stresses and ABA, SA treatments in seedlings. When exposed to cold stress, the CaNAC2 transcript had no obvious changes within $12 \mathrm{~h}$, significantly increased at $24 \mathrm{~h}$ and peaked up to eightfold at $48 \mathrm{~h}$ (Figure 3B). The transcript of CaNAC2 initiated to increase within $6 \mathrm{~h}$, and 


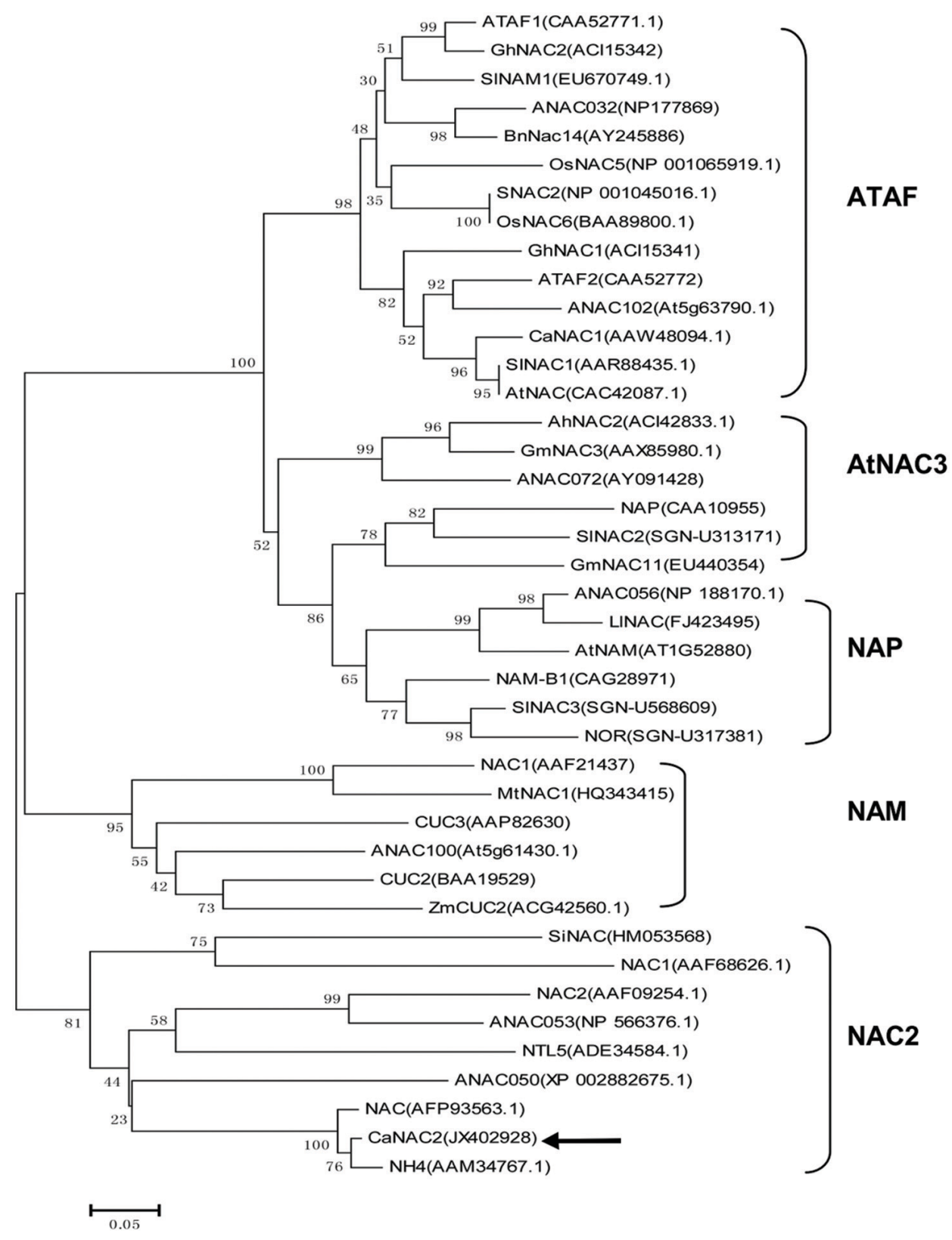

FIGURE 1 | A Neighbor-joining phylogenetic tree of CaNAC2 and NAC proteins from different plant species. The GenBank accession No. of NAC proteins are shown in the brackets. The CaNAC2 protein is marked with arrow. The NAC subgroup names are shown to the right of square.

reached the maximum after $12 \mathrm{~h}$ of salt stress (Figure 3C). Steady-state transcript of CaNAC2 reached to maximum after $48 \mathrm{~h}$ of $\mathrm{ABA}$ alone treatment (Figure 3E). Meanwhile, the expression of CaNAC2 was distinctly repressed by mannital and SA treatments (Figures 3D,F).

\section{CaNAC2-Silenced Pepper in Response to Chilling Stress and Salt Stress}

Compared to the negative control (inoculated with TRV2), the CaNAC2 silencing rate reached nearly $72 \%$ (Figure $4 \mathrm{~A}$ ), suggesting that VIGS was successful and effective for CaNAC2 gene silencing in pepper. About 35 silenced pepper seedlings were used for chilling stress, ABA pre-treatment followed by chilling stress or salt stress. Loss-of-function of $\mathrm{CaNAC2}$ in pepper plants increased susceptibility to chilling stress, the lipid peroxidation (MDA) content of the CaNAC2-silenced leaves was significantly higher than that of the empty vector control plants. Chilling stress after pretreated with $\mathrm{ABA}$, there is no significant difference between the negative control and the silenced pepper in the MDA content (Figure 4B). Obvious wilting appeared after $6 \mathrm{~h}$ of chilling stress in CaNAC2-silenced plants, while control leaves did not exhibit withering (Figure 4C). Responding to $300 \mathrm{mM}$ salt 


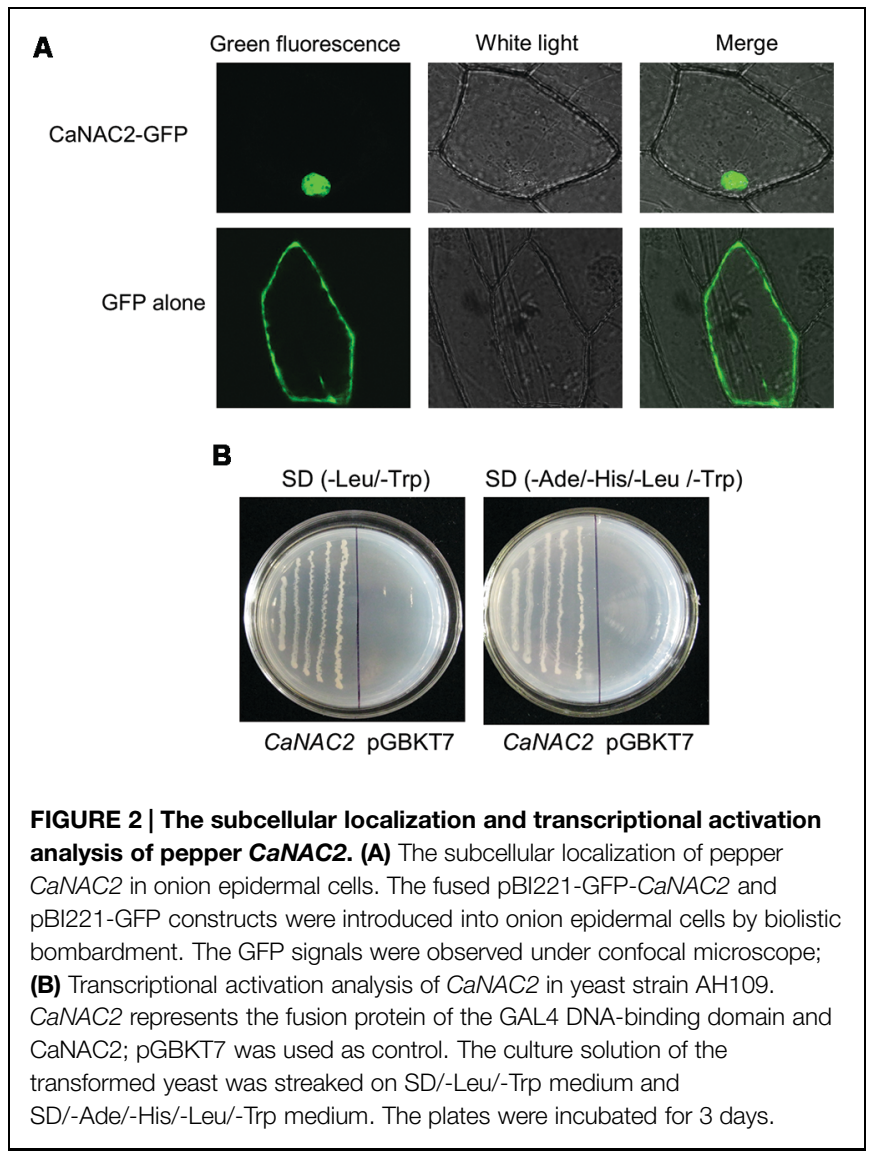

stress after $72 \mathrm{~h}$, the chlorophyll content of the CaNAC2-silenced leaf disks was significantly higher than that of the negative control plants (Figure 4D) and the leaf disks of CaNAC2-silenced pepper stayed green while the negative control appeared loss of chlorophyll (Figure 4E).

\section{Discussion}

In this study, a novel pepper NAC gene with full-length cDNA sequence, designated as CaNAC2, was isolated. The phylogenetic tree was constructed that CaNAC2 belonged to NAC2 subgroup (Figure 1) and further divided into the orthologous group $4 \mathrm{~d}$ (Ooka et al., 2003; Cenci et al., 2014). The C-terminal regions of other members from this group contained transmembrane motifs, such as SINAC, ANAC053, and TaNTL5 (Puranik et al., 2011; Lee et al., 2012; Tang et al., 2012). The TMpred program predicted that the C-terminal of CaNAC2 did not form transmembrane motifs, structurally distinct from NTLs (details not shown). This phylogenetic tree analysis indicated the extremely functional diversification of NAC gene subfamily in plant.

Sequence analysis predicted NLS at the N-terminus of CaNAC2 (Supplementary Figure S2). The subcellular localization assay demonstrated that the CaNAC2 was targeted to the nuclei (Figure 2A), the nuclei localization of which was different from NTLs of NAC2 subfamily. These results also proved that the C-terminal of CaNAC2 did not contain transmembrane motifs. Previous reports were shown that many NTLs proteins localized in cytoplasm and nucleus (Puranik et al., 2011; Lee et al., 2012; Tang et al., 2012). Results of transcription activation assays in yeast cells demonstrated that CaNAC2 encoded a NAC transcription activator, which had transcription activity (Figure 2B). The TRR of NAC TFs, generally lying at the highly diverged C-terminal, can activate transcription.

Tissue-specific transcription factor plays an important role in plant development and growth (He et al., 2005). In the present study, CaNAC2 was expressed in all tested tissues and high level in root and seed (Figure 3A), suggesting that CaNAC2 can play an important role in root growth and seed maturation. AtNAC2 was mainly expressed in root and can promote lateral root development (Xie et al., 2000). CarNAC1 was induced during seed development and germination (Peng et al., 2010). Many NAC family members have shown different responses to abiotic stress. For example, rice OsNAC6 was induced by low temperature, high salt, drought, and ABA treatment (Ohnishi et al., 2005; Nakashima et al., 2007), and over expression of this gene in rice can improve the tolerance of stress (Nakashima et al., 2007). BnNACs from Brassica campestris was also reported to be increased by multiple stresses such as wound, cold, and drought stress (Hegedus et al., 2003). Soybean nine NAC genes were induced by one or several treatments of drought, high salt, low temperature, or ABA (Tran et al., 2009). Furthermore, over expression of one of these genes (GmNAC20) enhanced salt and freezing tolerance in transgenic Arabidopsis plants (Hao et al., 2011). It has been reported that NAC transcription factors respond in both $\mathrm{ABA}$-dependent and -independent pathways to abiotic stress (Fujita et al., 2004; Tran et al., 2004). In this study, CaNAC2 was significantly induced by cold stress, salt, and ABA treatment (Figures 3B-E), indicating that this gene was involved in the response to cold and salt stress by ABA signaling. Meanwhile, CaNAC2 described here was strongly suppressed by osmotic stress (Figure 3D) and SA treatment (Figure 3F). Lipid peroxidation were more severely influenced by chilling stress in CaNAC2-silenced leaves compared with empty vector control plants (Figure 4B), indicating that silence of CaNAC2 in pepper plants increase sensitivity to cold stress. That result was consistent with the visible symptoms of leaf damage in CaNAC2silenced seedlings subjected to chilling stress (Figure 4C). In contrast, the leaf disks of CaNAC2-silenced pepper increased the chlorophyll content and appeared green under salt stress (Figures 4D,E). These results suggested that CaNAC2 is involved in the salt-induced chlorophyll degradation, which are different from that of the increased susceptibility to cold stress. The difference might be related to the imposed stress condition. Since different stresses may damage plant growth and development in specific ways, the plant could alleviate damage by different mechanisms.

In conclusions, CaNAC2 was highly expressed in seed and root. The expression of CaNAC2 was significantly induced by cold stress, salt, and ABA treatment and suppressed by osmotic stress and SA treatment. These findings suggested that CaNAC2 may be involved in response to various abiotic stresses. Silence 


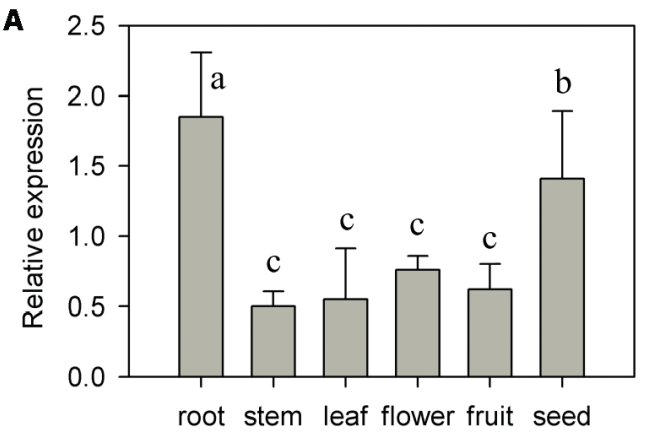

B
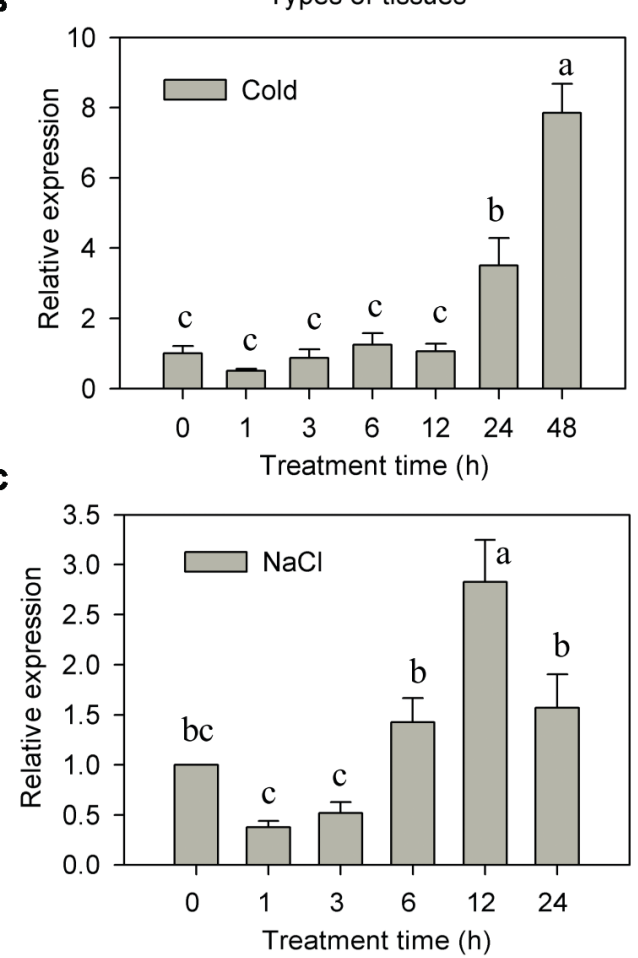
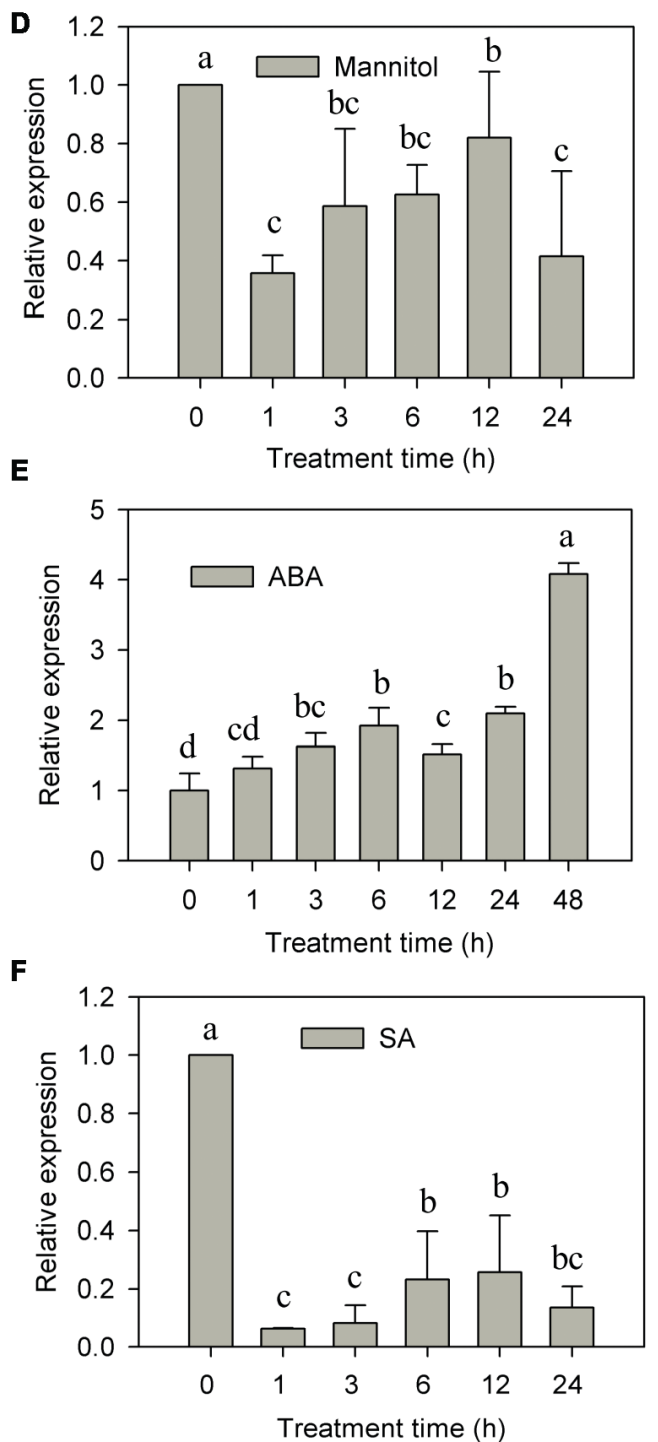

FIGURE 3 | CaNAC2 expression both in tissue and in response to stress and exogenous hormones. (A) Tissue specific expression of CaNAC2; (B) The pepper seedlings were exposed to cold stress $\left(6^{\circ} \mathrm{C}\right)$, (C) salt stress (300 mM NaCl) and (D) osmotic stress (300 mM mannitol) for the indicated times; the pepper seedlings were sprayed with exogenous ABA (E) and SA (F). Pepper CaUbi3 gene was used as an internal control for qRT-PCR. The transcript level of CaNAC2 at $\mathrm{O} \mathrm{h}$ is used as control (quantities of calibrator) and was assumed as 1. Results are the mean $\pm \mathrm{SE}$. Different letters indicate that the mean values are different by the Tukey HSD test $(p \leq 0.05)$. Each mean was compared with all the other mean values shown in the same figure.

of CaNAC2 led to increase susceptibility to cold stress and delayed the salt-induced leaf chlorophyll degradation, suggesting its possible role in stress tolerance. Currently, the authors are further studying the biological functions of CaNAC2 in response to abiotic stress using over expression method in pepper.

\section{Experimental Section}

\section{Plant Materials and Stress Treatments}

Pepper (C. annuum L.) cv P70 seeds were grown using a previously described method (Guo et al., 2012). The six-leaf seedlings were used to establish the following treatments. ABA and cold treatments were performed as described by Guo et al. (2013). For ABA and cold treatments, seedlings were sprayed with freshly prepared $0.57 \mathrm{mM} \mathrm{ABA}$ solution or water (control). At $72 \mathrm{~h}$ after foliar application, control and ABA treatment groups were subjected to cold stress at $6^{\circ} \mathrm{C}$. For salt and osmotic treatments, the seedling roots were immersed in solutions containing $300 \mathrm{mM}$ sodium chloride $(\mathrm{NaCl})$, or $300 \mathrm{mM}$ mannitol. For SA treatment, seedlings were sprayed with $5 \mathrm{mM}$ SA solution and maintained at $25^{\circ} \mathrm{C}$ for the indicated times. The treated seedlings were harvested after $0,1,3,6,12$, 24, and $48 \mathrm{~h}$ for examination of CaNAC2 expression pattern under various stress conditions. At each time point, two or three 
A

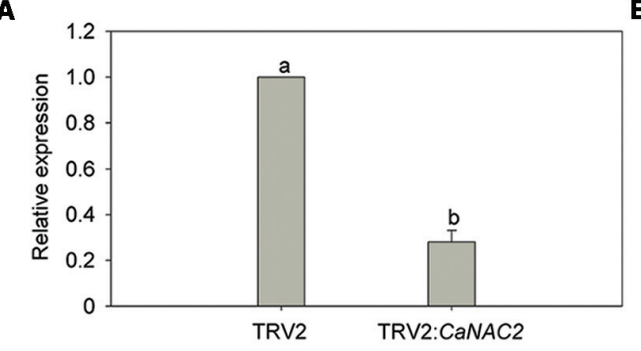

C

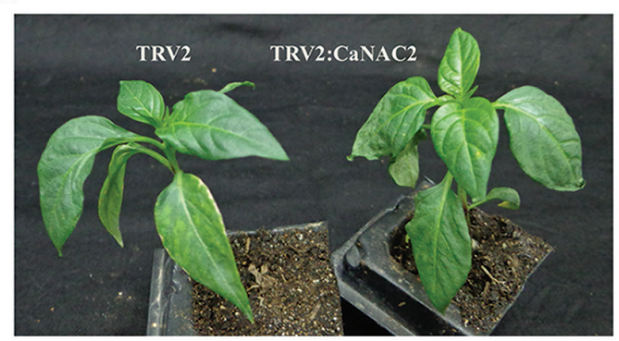

B

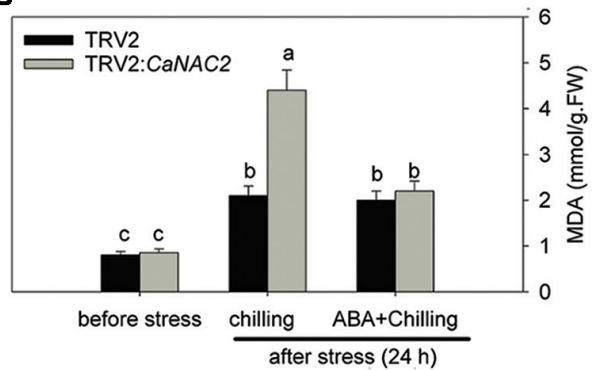

D

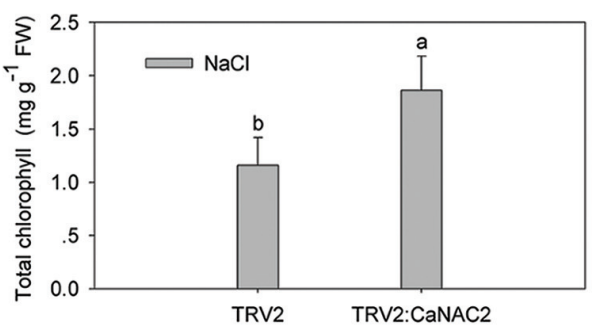

CK

$300 \mathrm{mM} \mathrm{NaCl}$

TRV2
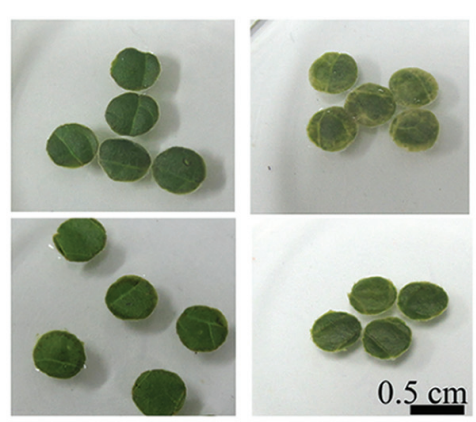

FIGURE 4 | CaNAC2-silenced pepper plants. (A) The expression of CaNAC2 in gene-silenced pepper (TRV2: CaNAC2) Cv P70 and control plants (TRV2:00) were tested at 45 days after inoculation; (B) Effect of ABA application and chilling stress on lipid peroxidation (MDA) content in CaNAC2-silenced pepper seedlings. (C) Phenotypes analysis of the CaNAC2-silenced and non-silenced pepper seedlings under $6^{\circ} \mathrm{C}$ cold stress for $6 \mathrm{~h}$. (D) Chlorophyll contents of leaf disks. (E) Leaf disks phenotypes. Leaf disks from the gene-silenced plants were floated in $300 \mathrm{mM} \mathrm{NaCl}$ solutions for $72 \mathrm{~h}$ at $25^{\circ} \mathrm{C}$. Bars with different lower case letters indicate significant differences compared to empty vector control leaves (Student's $t$-test, $p \leq 0.05$ ).

upper young leaves from four separate seedlings were collected to form one sample, wrapped with foil, immediately frozen in liquid nitrogen and stored at $-80^{\circ} \mathrm{C}$. The treatments were arranged in a

TABLE 1 | Primers used in this investigation.

\begin{tabular}{ll}
\hline Gene & Forward $(\mathbf{F})$ and Reverse $(\mathbf{R})$ primer $\mathbf{5}^{\prime} \longrightarrow \mathbf{3}^{\prime}$ \\
\hline RT-CaNAC2 & F: CCGACCTCTGACGTTGTTG \\
& R: AGTTCCTCAAGTCCTCGTC \\
GFP-CaNAC2 & F: GGAAATGGAGCAAGAAGGA \\
& R: CGGGGTACCACCATTCGGACGAGTTC \\
& (Kpnl digest site) \\
pGBKT7-CaNAC2 & F: TTCTCCTITATTATCTGTG \\
& R: GGTACAGGTTATAGCGAAACTACG \\
UBI-3 & F: TGTCCATCTGCTCTCTGTG \\
& R: CACCCCAAGCACAATAAGAC
\end{tabular}

randomized complete block design (RCBD) with three biological replicates.

\section{Isolation of CaNAC2 cDNA Clone and Sequence Analysis}

The NAC-homologous EST (GenBank accession No. JZ198750) isolated from a cold-related pepper seedling SSH library was reported by Guo et al. (2013). The full-length ORF of the NAC homolog was obtained using the cDNA fragment of this homolog as a probe by a homology-based candidate gene method (Guo et al., 2014). The full-length forward and reverse primers for CaNAC2 were 5'-TTCTTCCTTTTTATTTATCTGTG-3' and 5' GGTACAGGTTATAGCGAAA -CTACG-3', respectively.

The theoretical molecular weight $(\mathrm{Mw})$ and isoelectric point (pI) were calculated with the ExPASy compute pI/Mw tool (Bjellqvist et al., 1993). Sequence data were analyzed using Clustal W (Thompson et al., 1994). The phylogenetic 
tree was constructed using Mega5.0 by the neighbor-joining method. Homology searches in database were carried out using the default parameters of the BLAST program on the website http://www.ncbi.nlm.nih.gov/blast (Altschul et al., 1997).

\section{Real-Time Quantitative PCR (qRT-PCR) Analysis}

Gene-specific primers were designed using ProbeFinder Version $2.44^{1}$. Primer specificity was then confirmed by blasting each primer sequence against Phytozome $^{2}$ using BLASTN algorithm. RNA extraction, cDNA preparation, and qRT-PCR were performed as described by Guo et al. (2013). Total RNA was extracted using a TRIZOL reagent (Invitrogen, USA). DNA contamination was removed from the RNA samples using DNaseI RNase-free (50 U/ $\mu \mathrm{l}$, Fermentas). The quality and purity of the preparation were determined at $\mathrm{OD}_{260}: \mathrm{OD}_{280} \mathrm{~nm}$ absorption ratio (1.8-2.0) and the integrity of the preparations was ascertained by electrophoresis in a $1.2 \%$ agarose gel containing formaldehyde. And the first-strand cDNA synthesis was performed using the DNase-treated total RNA with a PrimeScript ${ }^{\mathrm{TM}}$ first-strand complementary DNA (cDNA) Synthesis Kit (TaKaRa, Japan). qRT-PCR was performed with an iCycler $\mathrm{iQ}^{\mathrm{TM}}$ Multicolor PCR Detection System (BioRad, Hercules, CA, USA). Relative gene expression levels were determined using the $2^{-\Delta \Delta C T}$ method. Total RNA was extracted from the leaves of pepper plants subjected to various stress for $0,1,3,6,12,24$, and $48 \mathrm{~h}$ as described above. The ubiquitin-conjugating protein gene (CaUbi3, GenBank accession No. AY486137.1) from pepper plants was amplified as a reference gene for normalization of CaNAC2 cDNA samples (Wan et al., 2011). The corresponding specific primers were listed in Table $\mathbf{1}$.

\section{Subcellular Localization Analysis of CaNAC2}

The ORFs of CaNAC2 cDNAs (without termination codon) were ligated into the pBI221-GFP vector, resulting in an inframe fusion protein of GFP gene and the CaNAC2 ORFs. The construct (pBI221-CaNAC2) and the control vector (pBI221 GFP) were transformed into onion epidermal cells by particle bombardment using a Biolistic PDS-1000/He gene gun system (Bio-Rad, Hercules, CA, USA). After $24 \mathrm{~h}$ at $25^{\circ} \mathrm{C}$ incubation of transformed onion epidermal cells, GFP signal was detected by a confocal fluorescence microscope (LSM510 Meta; Zeiss, Jena, Germany). The primers flanked with restriction sites for subcloning are listed in Table $\mathbf{1}$.

\section{Transcriptional Activation Analysis in Yeast}

To investigate the transcriptional activation, the CaNAC2 ORFs were fused in-frame with GAL4 DNA-binding domain in pGBKT7 to construct vectors. The pGBKT7 was used as a negative control. These different constructs were transformed into yeast strain AH109. The transformants were streaked on the selective medium lacking Leu and Trp (SD-Leu/-Trp) and

${ }^{1} \mathrm{http}: / /$ www.roche-applied-science.com

${ }^{2}$ http://www.phytozome.net/search.php? show $\$=\$$ blast selective medium lacking Trp, His, Leu, and Ade (SD-Ade/-His/Leu /-Trp) medium. After incubation at $28^{\circ} \mathrm{C}$ for $3-5$ days, the growth status of the transformants was evaluated. Primers for PCR amplification are provided in Table 1.

\section{VIGS Assay of CaNAC2 in Pepper Plants}

The TRV-based VIGS system was used for gene silencing, as described previously (Wang et al., 2013). To generate the CaNAC2/TRV2 construct, a 447-bp fragment of the CaNAC2 gene was PCR amplified from pepper. The resulting product was cloned into the TRV2 vector using the double digested method with enzymes of XbaI and BamHI. Forward primer $\quad\left(5^{\prime}\right.$-GCTCTAGACCGACCTCTGACGTTTGTTTG$\left.3^{\prime}\right)$ with XbaI enzyme site and reverse primer (5'-CG CGGATCCAGTTTCCTCAAGTCCTCG TTC-3') with BamHI enzyme site were used for vector construct. Agrobacterium tumefaciens strain GV3101 harboring pTRV1 was, respectively, mixed with pTRV2 (as the negative control), TRV2-CaPDS (as the positive control) or TRV2-CaNAC2 at a 1:1 ratio. The mixtures were inoculated into pepper cV P70 at the fully expanded cotyledons stage. After injection, all of the seedlings were placed at $18^{\circ} \mathrm{C}$ and $60 \%$ relative humidity for 2 days and then moved to a growth chamber according to the protocol. Leaf disks were excised from the gene-silenced pepper leaves using a cork borer $(0.5 \mathrm{~cm}$ in diameter $)$ and were floated in $300 \mathrm{mM} \mathrm{NaCl}$ solution at $25^{\circ} \mathrm{C}$ to test for salt stress. Total chlorophyll contents were spectrophotometrically measured after extracting into $80 \%$ (v/v) acetone (Guo et al., 2013). The level of lipid peroxidation was measured in terms of malondialdehyde (MDA), followed the procedure of Guo et al. (2012).

\section{Statistical Analyses}

Values were expressed as the mean \pm SE. The data were analyzed using analysis of variance (ANOVA), and the mean separation was analyzed using the Tukey Honestly Significant Differences (HSD) or Student's $t$-test $(p \leq 0.05)$ using the SPSS Base 12.0 for Windows (SPSS Inc., USA). A $p$-value $\leq 0.05$ was considered significant.

\section{Author Contributions}

W-LG. and R-GC conceived and designed the experiments; W-LG and B-HC performed the experiments; X-HD and Y-XY analyzed the data; Z-HG and S-BW contributed reagents/materials/analysis tools; $\mathrm{W}-\mathrm{LG}$ and $\mathrm{Y}-\mathrm{YZ}$ wrote the paper.

\section{Acknowledgments}

The following organizations are acknowledged for their financial support: National Natural Science Foundation of China (No. 31272163; 31201615), Jiangsu Agriculture Science and Technology Innovation Fund [CX(12)1004] and the Shaanxi Provincial Science and Technology Coordinating Innovative Engineering Project (2012KTCL02-09). 


\section{Supplementary Material}

The Supplementary Material for this article can be found online at: http://journal.frontiersin.org/article/10.3389/fpls.2015.00755

FIGURE S1 | The cDNA and putative amino acid sequences of pepper CaNAC2.

\section{References}

Altschul, S. F., Madden, T. L., Schaffer, A. A., Zhang, J., Zhang, Z., Miller, W., et al. (1997). Gapped BLAST and PSI-BLAST: a new generation of protein database search programs. Nucleic. Acids. Res. 25, 3389-3402.

Bjellqvist, B., Hughes, G. J., Pasquali, C., Paquet, N., Ravier, F., Sanchez, J. C., et al. (1993). The focusing positions of polypeptides in immobilized $\mathrm{pH}$ gradients can be predicted from their amino acid sequences. Electrophoresis 14, 1023-1031.

Cenci, A., Guignon, V., Roux, N., and Rouard, M. (2014). Genomic analysis of NAC transcription factors in banana (Musa acuminata) and definition of NAC orthologous groups for monocots and dicots. Plant Mol. Biol. 85, 63-80.

Collinge, M., and Boller, T. (2001). Differential induction of two potato genes, Stprx 2 and StNAC, in response to infection by Phytophthora infestans and to wounding. Plant Mol. Biol. 46, 521-529.

Deng, M. H., Wen, J. F., Zhu, H. S., and Zou, X. X. (2009). The hottest pepper variety in China. Genet. Resour. Crop Evol. 56, 605-608. doi: 10.1007/s10722009-9445-z

Duval, M., Hsieh, T. F., Kim, S. Y., and Thomas, T. L. (2002). Molecular characterization of AtNAM: a member of the Arabidopsis NAC domain superfamily. Plant Mol. Biol. 50, 237-248.

Ernst, H. A., Olsen, A. N., Larsen, S., and Lo Leggio, L. (2004). Structure of the conserved domain of ANAC, a member of the NAC family of transcription factors. EMBO Rep. 5, 297-303.

Fujita, M., Fujita, Y., Maruyama, K., Seki, M., Hiratsu, K., Ohme-Takagi, M., et al. (2004). A dehydration-induced NAC protein, RD26, is involved in a novel ABA-dependent stress-signaling pathway. Plant J. 39, 863-876.

Guo, M., Lu, J. P., Zhai, Y. F., Chai, W. G., Gong, Z. H., and Lu, M. H. (2015) Genome-wide analysis, expression profile of heat shock factor gene family (CaHsfs) and characterisation of CaHsfA2 in pepper (Capsicum annuum L.). BMC Plant Biol. 15:151. doi: 10.1186/s12870-015-0512-7

Guo, W. L., Chen, R. G., Du, X. H., Zhang, Z., Yin, Y. X., Gong, Z. H., et al. (2014). Reduced tolerance to abiotic stress in transgenic Arabidopsis overexpressing a Capsicum annuum multiprotein bridging factor 1. BMC Plant Biol. 14:138. doi: 10.1186/1471-2229-14-138

Guo, W. L., Chen, R. G., Gong, Z. H., Yin, Y. X., Ahmed, S. S., and He, Y. M. (2012). Exogenous abscisic acid increases antioxidant enzymes and related gene expression in pepper (Capsicum annuum) leaves subjected to chilling stress. Genet. Mol. Res. 11, 4063-4080.

Guo, W. L., Chen, R. G., Gong, Z. H., Yin, Y. X., and Li, D. W. (2013). Suppression subtractive hybridization analysis of genes regulated by application of exogenous abscisic acid in pepper plant (Capsicum annuum L.) leaves under chilling stress. PLoS ONE 8:e66667. doi: 10.1371/journal.pone.0066667

Han, Q., Zhang, J., Li, H., Luo, Z., Ziaf, K., Ouyang, B., et al. (2012). Identification and expression pattern of one stress-responsive NAC gene from Solanum lycopersicum. Mol. Biol. Rep. 39, 1713-1720.

Hao, Y. J., Wei, W., Song, Q. X., Chen, H. W., Zhang, Y. Q., Wang, F., et al. (2011). Soybean NAC transcription factors promote abiotic stress tolerance and lateral root formation in transgenic plants. Plant J. 68, 302-313.

He, X. J., Mu, R. L., Cao, W. H., Zhang, Z. G., Zhang, J. S., and Chen, S. Y. (2005). AtNAC2, a transcription factor downstream of ethylene and auxin signaling pathways, is involved in salt stress response and lateral root development. Plant J. 44, 903-916.

Hegedus, D., Yu, M., Baldwin, D., Gruber, M., Sharpe, A., Parkin, I., et al. (2003). Molecular characterization of Brassica napus NAC domain transcriptional activators induced in response to biotic and abiotic stress. Plant Mol. Biol. 53, 383-397.

Hu, H., Dai, M., Yao, J., Xiao, B., Li, X., Zhang, Q., et al. (2006). Overexpressing a NAM, ATAF, and CUC (NAC) transcription factor enhances drought
FIGURE S2 | Amino acid sequences alignment of pepper CaNAC2 with others. The five conserved motifs ( $A$ to $E$ ) are shown by thin underlines. The rich Ser regions in the C-terminal part are shown by thick underlines. The nuclear localization signal is lined with double arrows. The genes included are ANAC050 (XP_002882675.1), ANAC051/52 (XP_002882676.1), ANAC053 (NP_566376.1), ANAC078 (At5g04410), and NAC2 (AAF09254.1) from Arabidopsis, NH4 (AAM34767.1) from Petunia.

resistance and salt tolerance in rice. Proc. Natl. Acad. Sci. U.S.A. 103, 12987-12992.

Hu, H., You, J., Fang, Y., Zhu, X., Qi, Z., and Xiong, L. (2008). Characterization of transcription factor gene SNAC2 conferring cold and salt tolerance in rice. Plant Mol. Biol. 67, 169-181.

Jeong, J. S., Kim, Y. S., Baek, K. H., Jung, H., Ha, S. H., Do Choi, Y., et al. (2010). Root-Specific expression of osnac10 improves drought tolerance and grain yield in rice under field drought conditions. Plant Physiol. 153, 185-197.

Kim, S. Y., Kim, S. G., Kim, Y. S., Seo, P. J., Bae, M., Yoon, H.-K., et al. (2007). Exploring membrane-associated NAC transcription factors in Arabidopsis: implications for membrane biology in genome regulation. Nucleic. Acids. Res. 35, 203-213.

Korkmaz, A., Korkmaz, Y., and Demirkiran, A. R. (2010). Enhancing chilling stress tolerance of pepper seedlings by exogenous application of 5-aminolevulinic acid. Environ. Exp. Bot. 67, 495-501. doi: 10.1016/j.envexpbot.2009. 07.009

Lee, S., Seo, P. J., Lee, H. J., and Park, C. M. (2012). A NAC transcription factor NTL4 promotes reactive oxygen species production during drought-induced leaf senescence in Arabidopsis. Plant J. 70, 831-844.

Nakashima, K., Tran, L. S., Van Nguyen, D., Fujita, M., Maruyama, K., Todaka, D. et al. (2007). Functional analysis of a NAC-type transcription factor OsNAC6 involved in abiotic and biotic stress-responsive gene expression in rice. Plant J. $51,617-630$.

Oh, S. K., Lee, S., Yu, S. H., and Choi, D. (2005). Expression of a novel NAC domain-containing transcription factor (CaNAC1) is preferentially associated with incompatible interactions between chili pepper and pathogens. Planta 222, 876-887.

Ohnishi, T., Sugahara, S., Yamada, T., Kikuchi, K., Yoshiba, Y., Hirano, H. Y., et al. (2005). OsNAC6, a member of the NAC gene family, is induced by various stresses in rice. Genes. Genet. Syst. 80, 135-139.

Olsen, A. N., Ernst, H. A., Leggio, L. L., and Skriver, K. (2005). NAC transcription factors: structurally distinct, functionally diverse. Trends Plant Sci. 10, 79-87.

Ooka, H., Satoh, K., Doi, K. Nagata, T., Otomo, Y., Murakami, K., et al. (2003). Comprehensive analysis of NAC family genes in Oryza sativa and Arabidopsis thaliana. DNA Res. 10, 239-247.

Peng, H., Yu, X., Cheng, H., Shi, Q., Zhang, H., Li, J., et al. (2010). Cloning and characterization of a novel NAC family gene CarNAC1 from chickpea (Cicer arietinum L.). Mol. Biotechnol. 44, 30-40.

Pressman, E., Shaked, R., and Firon, N. (2006). Exposing pepper plants to high day temperatures prevents the adverse low night temperature symptoms. Physiol. Plant. 126, 618-626.

Puranik, S., Bahadur, R. P., Srivastava, P. S., and Prasad, M. (2011). Molecular cloning and characterization of a membrane associated NAC family gene, SiNAC from foxtail millet [Setaria italica (L.) P. Beauv]. Mol. Biotechnol. 49, $138-150$.

Schwarz, D., Rouphael, Y., Colla, G., and Venema, J. H. (2010). Grafting as a tool to improve tolerance of vegetables to abiotic stresses: thermal stress, water stress and organic pollutants. Sci. Hortic. 127, 162-171. doi: 10.1016/j.scienta.2010.09.016

Shinozaki, K., Yamaguchi-Shinozaki, K., and Seki, M. (2003). Regulatory network of gene expression in the drought and cold stress responses. Curr. Opin. Plant Biol. 6, 410-417. doi: 10.1016/S1369-5266(03)00092-X

Tang, Y., Liu, M., Gao, S., Zhang, Z., Zhao, X., Zhao, C., et al. (2012). Molecular characterization of novel TaNAC genes in wheat and overexpression of TaNAC2a confers drought tolerance in tobacco. Physiol. Plant. 144, 210-224.

Thomashow, M. F. (1999). Plant cold acclimation: freezing tolerance genes and regulatory mechanisms. Annu. Rev. Plant Physiol. Plant Mol. Biol. 50, 571-599. doi: 10.1146/annurev.arplant.50.1.571 
Thompson, J. D., Higgins, D. G., and Gibson, T. J. (1994). CLUSTAL W: improving the sensitivity of progressive multiple sequence alignment through sequence weighting, position-specific gap penalties and weight matrix choice. Nucleic. Acids. Res. 22, 4673-4680.

Tran, L. S., Nakashima, K., Sakuma, Y., Simpson, S. D., Fujita, Y., Maruyama, K., et al. (2004). Isolation and functional analysis of Arabidopsis stress-inducible NAC transcription factors that bind to a drought-responsive cis-element in the early responsive to dehydration stress 1 promoter. Plant Cell 16, 2481-2498.

Tran, L. S., Quach, T. N., Guttikonda, S. K., Aldrich, D. L., Kumar, R., Neelakandan, A., et al. (2009). Molecular characterization of stress-inducible GmNAC genes in soybean. Mol. Genet. Genomics 281, 647-664.

Wan, H., Yuan, W., Ruan, M., Ye, Q., Wang, R., Li, Z., et al. (2011). Identification of reference genes for reverse transcription quantitative real-time PCR normalization in pepper (Capsicum annuum L.). Biochem. Biophys. Res. Commun. 416, 24-30. doi: 10.1016/j.bbrc.2011.10.105

Wang, J. E., Li, D. W., Gong, Z. H., and Zhang, Y. L. (2013). Optimization of virusinduced gene silencing in pepper (Capsicum annuum L.). Genet. Mol. Res. 12, 2492-2506.
Xie, Q., Frugis, G., Colgan, D., and Chua, N. H. (2000). Arabidopsis NAC1 transduces auxin signal downstream of TIR1 to promote lateral root development. Genes Dev. 14, 3024-3036. doi: 10.1101/gad.852200

Yang, R., Deng, C., Ouyang, B., and Ye, Z. (2011). Molecular analysis of two saltresponsive NAC-family genes and their expression analysis in tomato. Mol. Biol. Rep. 38, 857-863.

Conflict of Interest Statement: The authors declare that the research was conducted in the absence of any commercial or financial relationships that could be construed as a potential conflict of interest.

Copyright (c) 2015 Guo, Wang, Chen, Chen, Du, Yin, Gong and Zhang. This is an open-access article distributed under the terms of the Creative Commons Attribution License (CC BY). The use, distribution or reproduction in other forums is permitted, provided the original author(s) or licensor are credited and that the original publication in this journal is cited, in accordance with accepted academic practice. No use, distribution or reproduction is permitted which does not comply with these terms. 